\title{
Cracks Cleave Crystals
}

\author{
M. Marder \\ Center for Nonlinear Dynamics and Department of Physics \\ The University of Texas at Austin, Austin TX 78712
}

\begin{abstract}
The problem of finding what direction cracks should move is not completely solved. A commonly accepted way to predict crack directions is by computing the density of elastic potential energy stored well away from the crack tip, and finding a direction of crack motion to maximize the consumption of this energy. I provide here a specific case where this rule fails. The example is of a crack in a crystal. It fractures along a crystal plane, rather than in the direction normally predicted to release the most energy. Thus, a correct equation of motion for brittle cracks must take into account both energy flows that are described in conventional continuum theories and details of the environment near the tip that are not.
\end{abstract}

\section{INTRODUCTION}

Much of the continuum theory of fracture concerns itself with the initiation of cracks, and their speed in response to varying loads [1]. Cracks also choose a direction in which to move. The continuum theory of fracture has no law to describe unambiguously which way they choose. However, there is a rule that is widely employed in practice to calculate the direction of crack motion. This rule is the principle of local symmetry. It says that cracks advance in the direction such that shear stresses on the faces of the crack vanish near the tip; the stresses are purely tensile, and pull the crack faces apart. Equivalently, cracks move in a direction that maximizes the consumption of energy stored in linear elastic fields in front of the tip. The rule was first proposed for slowly moving cracks by Goldstein and Salganik[2], generalized to rapidly moving cracks by Adda-Bedia, Arias, Ben Amar and Lund[3], and has recently been derived carefully from a variational principle by Oleaga[4].

Experimental checks are not numerous, but they confirm the principle of local symmetry. They have been carried out in amorphous materials such as glass where alternatives are difficult to imagine[5, 6, 7]. Experiments in crystals, and the art of cutting gems [8], find a tendency of cracks to travel along special atomic planes[9, 10]. However, the crystals where these experiments have been performed are macroscopically anisotropic. The preference of cracks for certain directions can be attributed to the lack of isotropy in the continuum theory. Thus it has been reasonable to believe that cracks in a macroscopically homogeneous and isotropic material should always move in accord with the dictates of local symmetry.

I will provide here a specific system where the principle of local symmetry is not obeyed, despite the fact that macroscopically the system is homogeneous and isotropic. The demonstration comes from combined analytical and numerical work. The numerical computations involve small numbers of atoms $(40,000)$. However, by combining the computations with scaling theory, one can predict the outcome of experiments with arbitrarily large numbers of atoms, and over arbitrarily large time intervals[11].

\section{IDEAL BRITTLE CRYSTAL}

The material in which I will investigate crack motion is an ideal brittle triangular crystal with equilibrium lattice spacing $a$ in which atoms obey the equation of motion

$$
m \ddot{\vec{u}}_{i}=\sum_{j}\left[\vec{f}\left(\vec{u}_{j i}\right)+\vec{g}\left(\dot{\vec{u}}_{j i}, \vec{u}_{j i}\right)\right]
$$

with $\vec{u}_{j i} \equiv \vec{u}_{j}-\vec{u}_{i}$. The functions $\vec{f}$ and $\vec{g}$ have the specific forms

$$
\vec{f}(\vec{r})=\kappa \hat{r}(r-a) \theta\left(r_{c}-r\right) ; \quad \vec{g}(\dot{\vec{r}}, \vec{r})=\beta \dot{\vec{r}} \theta\left(r_{c}-r\right) .
$$

Atoms interact with a central force $f$ that varies linearly around the equilibrium spacing of length $a$, and whose scale is set by $\kappa$. If the distance between atoms increases to more than $r_{c}$, the force drops abruptly to zero. In addition, atoms experience Kelvin dissipation $g$; its scale is set by $\beta$, is proportional to the relative velocities of neighbors, and also drops to zero when the distance between neighbors exceeds $r_{c}$. The macroscopic elastic theory of this crystal is homogeneous and isotropic, with Young's modulus $Y=(5 \sqrt{3} / 4)(\kappa / a)$ and Poisson ratio $\nu=1 / 4$.

I will consider systems that are an even number $N$ atomic planes high. For cracks to run through such crystals, they must be under tension. The theory of fracture [1] says that the tension must be great enough ahead of the tip so that the energy stored in a vertical slice one unit cell wide is enough to snap a pair of bonds at the crack tip (the Griffith criterion). Bonds snap when 


\section{Upper row of atoms held rigid}
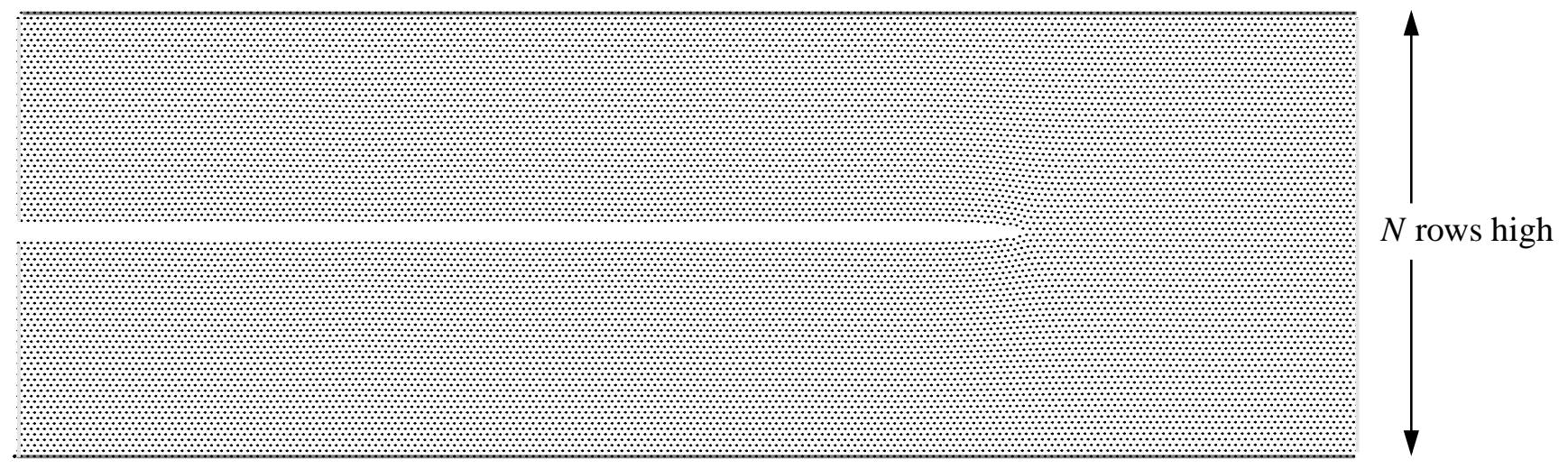

Lower row of atoms held rigid

FIG. 1: Setting for numerical experiment to find steady crack states. Atoms are originally arrayed in triangular lattice 80 rows high, and three times as long as it is tall. Primitive vectors for the equilibrium lattice are $a\left(\begin{array}{ll}1 & 0\end{array}\right)$ and $a(1 / 2 \sqrt{3} / 2)$. The crack tip is defined as the location of the rightmost atom whose nearest vertical neighbor is at distance greater than $2.5 a$. When the crack tip approaches within $60 a$ of the right boundary, 10 columns of new crystal are attached to the right boundary, and the same amount discarded from the left hand side. In the discussion leading to Eq. 7), top and bottom rows of atoms are held rigid and stretched vertically apart by a distance $\delta_{y}$. To produce Figure 3 the top boundary is also slid horizontally relative to the bottom by an amount $\delta_{x}$.

they are stretched beyond their original length by an amount $r_{c}-a$, so the elastic energy stored in a vertical strip of material of horizontal length $a$ needs to be at least

$$
\text { 2(two bonds per node) } \times \frac{1}{2} \times \kappa\left(r_{c}-a\right)^{2}=\kappa\left(r_{c}-a\right)^{2} .
$$

There are $N-1$ rows of slanted bonds. When they are stretched vertically by a small distance $\epsilon_{y}$, the length of each bond increases to linear order by an amount $\epsilon_{r}=(\sqrt{3} / 2) \epsilon_{y}$. Therefore, at the Griffith energy, the total energy stored per unit length is

$$
\begin{aligned}
& 2(\text { two bonds per site }) \times(N-1) \times \frac{1}{2} \times \kappa\left(\epsilon_{r}\right)^{2}=\kappa\left(r_{c}-a\right)^{2} \\
& \Rightarrow \epsilon_{r}=\left(r_{c}-a\right) / \sqrt{N-1} \Rightarrow \epsilon_{y}=\frac{2}{\sqrt{3}}\left(r_{c}-a\right) / \sqrt{N-1}
\end{aligned}
$$

Then for fracture first to be energetically possible, one rigidly raises the top of the crystal above its equilibrium position by a vertical distance $y_{c}$

$$
y_{c}=\frac{2}{\sqrt{3}}\left(r_{c}-a\right) \sqrt{N-1}
$$

In the limit $r_{c} \rightarrow a$ steady state cracks in this crystal are described by exact analytical solutions [12, 13, 14, 15]. The most important observation to extract from these solutions is that the natural dimensionless measure of how much one has loaded the crystal is obtained by rigidly displacing its upper surface a distance $\delta_{y}$ and then forming the ratio

$$
\Delta \equiv \delta_{y} / y_{c} .
$$

That is, $\Delta$ is a variable proportional to the strain applied far ahead of the crack. It equals 1 when the crystal has been loaded precisely to the Griffith point where fracture first becomes possible. The analytical solutions demonstrate that if one measures crack speed $v$ and plots it as a function of loading $\Delta$, the results become independent of system height $N$ to better than $1 \%$ for surprisingly small values of $N$, on the order of $N=50$, as shown in Figure 2 This statement is true so long as the crack speed is not too large. Continuum theory predicts that cracks in tension cannot exceed the Rayleigh wave speed $c_{R}$, which for this model equals .563 $\sqrt{\kappa a^{2} / m}$. Figure 2 shows that crack speed is practically independent of system height for $N>50$ and $v<.9 c_{R}$.

The model Eq. (1) is more realistic when $r_{c}=1.2 a$ than in the limit $r_{c} \rightarrow a$, since atomic bonds in real brittle materials acutally give way when extended by about 20\%. For $r_{c}=1.2$, analytical techniques are no longer available to provide exact solutions. However, the scaling properties provided by the analytical solutions continue to hold. The relationship between crack speed $v$ 


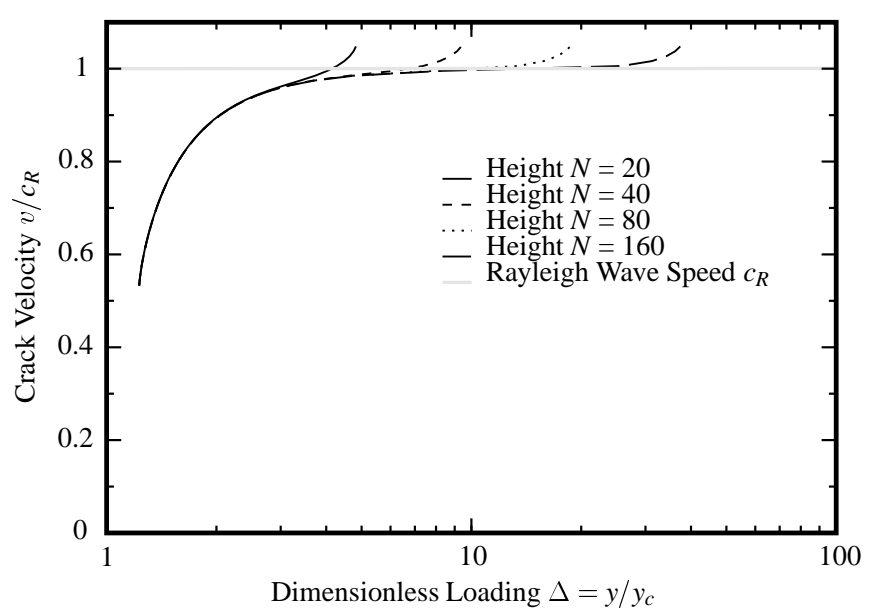

FIG. 2: Relationship between velocity $v$ and dimensionless loading $\Delta$ for lattice strips of varying height $N$ in pure tension. The calculations are performed in the limit $r_{c} \rightarrow a$ with Kelvin dissipation $\beta=0.01$ with the Wiener-Hopf technique [12, 13, 14]. The left-hand portions of the curve are almost completely independent of system height. The cracks are presumed to travel along a weak interface that precludes transverse instabilities, and therefore the curves continue up and through the Rayleigh wave speed $c_{R}$. Were the curves to be terminated at the points where cracks become unstable in homogeneous crystals, they would be nearly indistinguishable.

(A)
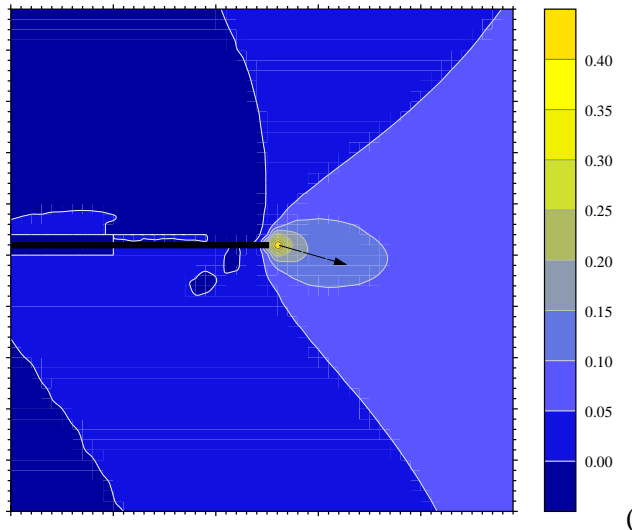

(B)

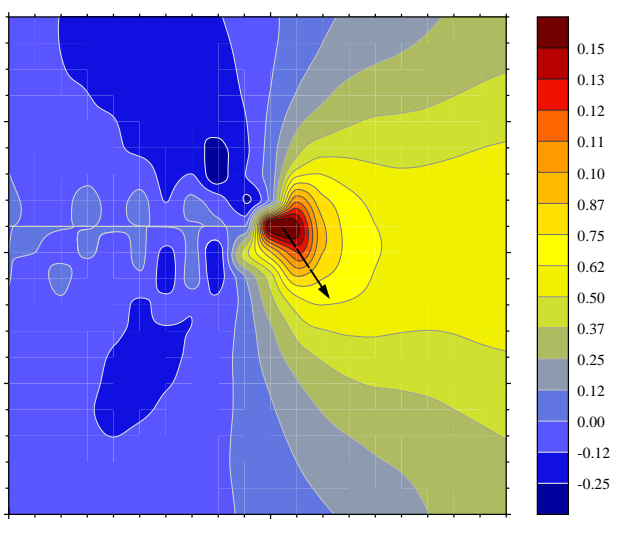

FIG. 3: Color contour plots of tensile stress field $\sigma_{\theta \theta}$ surrounding crack tips in strips with rigid vertical tensile $\left(\delta_{y}\right)$ and horizontal shear $\left(\delta_{x}\right)$ displacements of upper and lower boundaries, computed from solutions of Eq. 1 Arrows show directions the cracks should turn according to local symmetry. Instead, they travel stably forever along the horizontal axis. The circular stress islands to the left of the crack result from averaging over high-frequency waves emitted by the crack and traveling left to right. (A) System $N=150$ rows high, $\delta_{y} / y_{c}=1.29$, $\delta_{x} / \delta_{y}=.23, r_{c}=1.2$, force constant $\kappa=1$, and Kelvin dissipation $\beta=2$, resulting in a crack velocity $v / c_{R}=.01$. (B) As in (A), but $N=200$ rows high and Kelvin dissipation $\beta=.02$, resulting in a crack velocity $v / c_{R}=.83$. The smaller value of $\beta$ is completely responsible for the larger crack speed. The larger system is chosen because details of the fast-moving crack are more difficult to resolve.

and loading $\Delta$ is practically independent of system height $N$ once $N$ reaches a value of around 50 . As a result one can accurately predict the relationship between crack velocity $v$ and loading $\Delta$ up to the macroscopic limit by performing computations in systems of microscopic dimensions.

Having established that microscopic computations have a legitimate macroscopic interpretation, I will now set out to show that the principle of local symmetry does not always correctly predict crack paths. Instead there is an interplay between the direction preferred by far-field stresses, and the direction preferred by microstructure. This conclusion comes from seeding cracks on the centerline of strips as in Figure 1 but then loading them with a mixture of tension and shear. The top and bottom boundaries are displaced vertically by distance $\delta_{y}$ and horizontally by distance $\delta_{x}$.

The main result is represented in Figure 3. This figure shows contours of the tensile opening stress surrounding crack tips moving at two different speeds in a crystal. The contours are tilted away from the horizontal axis. The principle of local symmetry predicts that a crack tip surrounded by such stress fields should rapidly turn and move toward the tip of the largest 
(A)

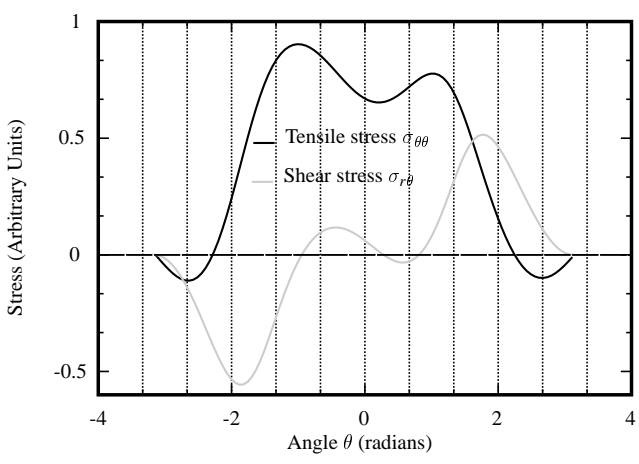

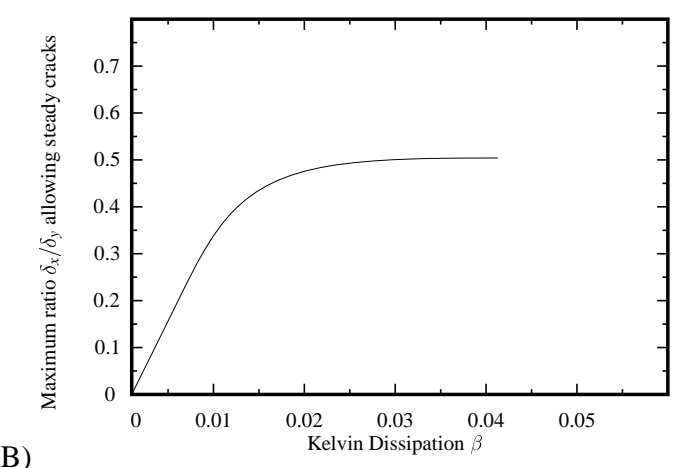

(B)

FIG. 4: (A) Theoretical asymptotic tensile stress $\sigma_{\theta \theta}(\theta)$ and shear stress $\sigma_{r \theta}(\theta)$ around crack tip for crack traveling at speed $v / c_{R}=.83$ under the mixed mode loading applied to the crack in Figure 3 computed with continuum techniques of [17]. (B). (B) Diagram showing combinations of Kelvin dissipation and mixed-mode loading that permit steady motion down center of strip from solutions of Eq. 1 . The simulations begin with purely tensile loading at $\Delta=1.29,\left(\delta_{x}=0\right)$ and then increase the horizontal displacement of the upper boundary $\delta_{x}$ while keeping the vertical displacement $\delta_{y}$ fixed until steady motion becomes unstable.

lobe. However, the cracks move steadily and stably along the horizontal axis forever. These cracks follow crystal planes, not external stress fields. It should be emphasized that the macroscopic elastic properties of a triangular crystal are completely isotropic. Only the presence of atomic-scale planes can explain the failure of the cracks to follow the directions predicted by local symmetry.

To check the principle of local symmetry, it is necessary to compute the continuum elastic fields surrounding these cracks. This task has been performed in two ways, which agree. First, the elastic stress fields were computed directly from the positions of atoms in the simulation by taking binned spatial averages in volumes $V_{0}$ of $\sigma_{\alpha \beta}=\left(1 / 4 V_{0}\right) \sum_{j}\left[r_{i j}^{\alpha} f_{i j}^{\beta}+r_{i j}^{\beta} f_{i j}^{\alpha}\right]$, where $\vec{f}_{i j}$ is the force between atoms $i$ and $j[16]$. Second, the system was viewed as a fracture in a continuous elastic strip, and the stress fields around the crack tip were computed exactly with techniques of fracture mechanics[17] (Figure 4(A)).

The cracks in Figure 3 travel in lattices where the upper boundary is rigidly displaced by amounts $\left(\delta_{x}, \delta_{y}\right)$, where $\delta_{x} / \delta_{y}=$ $\tan (.073 \pi)$. Fracture mechanics calculation predicts that tensile stresses are maximal at angles of $\theta=-16^{\circ}($ case $(\mathrm{A}))$ and $\theta=-57^{\circ}$ (case (B)) to the $x$ axis, as shown in Figure 4 Therefore, the principle of local symmetry predicts that the crack in (A) should quickly turn and travel at an angle of $-16^{\circ}$, and the crack in (B) should quickly turn along an angle of $-57^{\circ}$. The arrows in Figure 3 show these directions of crack motion as predicted by the principle of local symmetry. Stress contours, computed directly from spatial averages over interatomic forces, indeed have lobes in the directions continuum theory predicts. But the cracks do not move in these directions. Instead, they travel endlessly along the crystal planes defined by the $x$ axis.

How much shear loading can be placed upon a strip before horizontally traveling cracks become unstable? An answer to this question is contained in Figure 4(B) The results depend upon the strength of Kelvin dissipation ( $\beta$ in Eq. (2)). Without any Kelvin dissipation, cracks even under purely tensile loading are unstable. As $\beta$ increases, the range of shear loading cracks can withstand while running along a crystal plane also increases. Physically, Kelvin dissipation has this effect because it damps the motion of atoms in the vicinity of the tip. A crack can only begin to depart from the $x$ axis by breaking a first horizontal bond above the main crack line. Breaking such a bond is easiest when atoms in the vicinity of the tip oscillate with large amplitude as the tip passes. Kelvin dissipation damps motion of atoms near the tip, and makes it more stable.

\section{CONTINUUM THEORY REVISITED}

The principle of local symmetry does not explain the results depicted in Figure 3 However, perhaps a simple modification of the usual rule could do so. In a crystal, the energy per area $\Gamma\left(\theta_{c}\right)$ a crack needs to create new surfaces depends upon the angle $\theta_{c}$ between the crack and crystalline planes. In an amorphous material, there should be no such angular dependence of surface energy. This observation suggests modifying the principle of local symmetry to choose a crack direction that maximizes the difference between energy flowing to the crack tip along $\theta_{c}$ and energy required along $\theta_{c}$ to create new surfaces.

By returning to the derivations of [3] and [4] I have found a formal procedure to generalize the principle of local symmetry in this fashion. This generalization does capture the tendency of cracks in crystals to move along crystal planes. However, it does not explain quantitatively the results in Figures 3 and 4 For example, I could not find a way within continuum theory to include the effects of Kelvin dissipation $\beta$, since Kelvin dissipation is forbidden in continuum fracture mechanics[18].

Ordinarily in fracture mechanics, crack motion is determined by a scalar energy flux $G$, which describes the energy per length a crack can extract from elastic fields by moving forward unit distance. Adda-Bedia et al. [3] and Oleaga[4] define an energy flux 


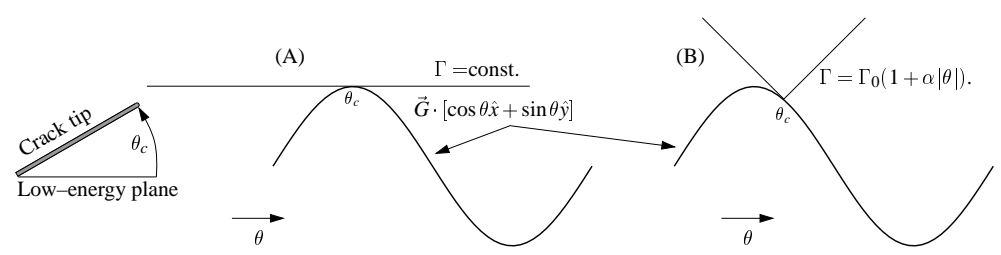

FIG. 5: Graphical representations of Eqs. 8. Crack directions are given by moving the upper curve (representing fracture energy versus angle) down until it first touches the lower curve (representing energy flux to crack). The contact point of the two curves gives the direction of crack motion $\theta_{c}$. (A) When fracture energy is independent of direction, contact can only occur at the maximum of the lower curve. This means that $\vec{G}$ is parallel to the direction of crack motion $\theta_{c}$. (B) If fracture energy has a cusp-like minimum, there are many ways to satisfy Eqs. (8). For some, the upper tip just touches the lower curve; for these, the crack cleaves a plane at $\theta_{c}=0$. For others, $\theta_{c}$ varies continuously away from 0 . A similar graphical construction, suggested by experimental data, is found in [10].

vector $\vec{G}$. The component of this vector parallel to the crack tip is the energy flux $G_{\|}=G$. The perpendicular component $G_{\perp}$ gives the energy that would come to the crack tip if it could somehow be slid upwards normal to its current direction. According to Oleaga[4], crack motion obeys two rules. Let a low-energy plane lie along the $\hat{x}$ axis at $\theta=0$, and let the current angle of the crack tip relative to this axis be $\theta_{c}$. First, the crack chooses direction and speed so that the energy per area $\Gamma$ needed to create new surface equals the energy per area brought to the tip:

$$
\Gamma\left(\theta_{c}\right)=G=\vec{G} \cdot\left[\cos \theta_{c} \hat{x}+\sin \theta_{c} \hat{y}\right] .
$$

Second, the crack direction $\theta_{c}$ is chosen by the condition that no other angle $\theta$ provides enough energy for the crack to move:

$$
\Gamma(\theta) \geq \vec{G} \cdot[\cos \theta \hat{x}+\sin \theta \hat{y}] .
$$

The energy flux vector $\vec{G}$ depends upon the crack speed, the current direction of the crack tip $\theta_{c}$, and the asymptotic stress field approaching the tip, but not upon angle $\theta$; detailed expressions for each component are given by Eqs. (4.5) and (4.6) in Ref. [4].

When the fracture energy $\Gamma$ is independent of direction, these rules imply the familiar principle of local symmetry. To see why, note that if $\vec{G}$ does not point along $\theta_{c}$ there must be some value of $\theta$ that will violate the inequality in $(8 \mathrm{~b}$, as shown in Figure 5 (A). However, if $\Gamma(\theta)$ is not constant, matters are not so simple. In particular, suppose that $\Gamma$ has the dependence expected for small angles $\theta$ in the presence of crystal planes $\Gamma(\theta)=\Gamma_{0}(1+\alpha|\theta|)$, where $\alpha>0$ is a constant of order unity and $\Gamma_{0}$ is the fracture energy along the plane. Eqs. [8] now have the graphical interpretation shown in Figure [5](B). The crack direction is determined by sliding a sharp tip over a sine curve. The tip must just touch the sine curve at one point, and can never dip below it. There are two sorts of solutions. In the first, contact occurs right at the tip where $\theta_{c}=0$, and the crack cleaves the low-energy plane. Such solutions can occur even if $\vec{G}$ does not point along $\theta=0$, thus violating the customary principle of local symmetry. A second sort of solution is possible if the slope of the sine curve somewhere becomes larger than $\alpha$; for these, the crack can travel at an angle different from $\theta=0$.

How well does this generalized principle of local symmetry work? Qualitatively, it has many sensible features. It correctly predicts that cracks can be trapped on crystal planes, and run along them for a range of loading conditions. It predicts that beyond a critical loading, the crack will stop following the crystal plane. Quantitatively, I have been unable to reproduce the results of microscopic calculations using Eqs. (8). In particular, Eqs. (8) predict that all the cracks described in Figure 4)(B) should travel stably along the $x$ axis, and misses the correct result which is that at a certain value of shear loading these cracks are no longer stable.

Therefore, I do not believe there is a substitute for detailed microscopic analysis if one wants to treat correctly the dynamics of cracks in brittle materials.

\section{Acknowledgments}

The National Science Foundation (DMR-9877044 and DMR-0101030) supported this work. Matt Lane provided many comments to help me improve this manuscript.

[1] K. B. Broberg, Cracks and Fracture. San Diego: Academic Press, 1999. 
[2] R. V. Goldstein and R. Salganik, "Brittle fracture of solids with arbitrary cracks," International Journal of Fracture, vol. 10, pp. 507-523, 1974.

[3] M. Adda-Bedia, M. Arias, M. B. Amar, and F. Lund, "Generalized Griffith criterion for dynamical fracture and the stability of crack motion at high velocities," Physical Review E, vol. 60, pp. 2366-2376, 1999.

[4] G. E. Oleaga, "Remarks on a basic law for dynamic crack propagation," Journal of the Mechanics and Physics of Solids, vol. 49, pp. 2273-2306, 2001.

[5] A. Yuse and M. Sano, "Transition between crack patterns in quenched glass plates," Nature, vol. 362, pp. 329-31, 1993.

[6] M. Adda-Bedia and Y. Pomeau, "Crack instabilities in a heated glass strip," Physical Review E, vol. 52, pp. 4105-4113, 1995.

[7] K. Ravi-Chandar and B. Yang, "On the role of microcracks in the dynamic fracture of brittle materials," Journal of the Mechanics and Physics of Solids, vol. 45, pp. 535-563, 1997.

[8] J. Field, "Brittle fracture: its study and application," Contemporary Physics, vol. 12, pp. 1-31, 1971.

[9] D. Sherman and I. Be'ery, "Fracture mechanisms of sapphire under bending," Journal of Materials Science, vol. 35, no. 5, pp. 1283-93, 2000.

[10] R. D. Deegan, S. Chheda, L. Patel, M. Marder, H. L. Swinney, J. Kim, and A. de Lozanne, "Wavy and rough cracks in silicon,” Physical Review E, vol. 67, p. 066209, 2003.

[11] J. Fineberg and M. Marder, "Instability in dynamic fracture," Physics Reports, vol. 313, pp. 1-108, 1999.

[12] L. Slepyan, "Dynamics of a crack in a lattice," Soviet Physics Doklady, vol. 26, pp. 538-540, 1981.

[13] L. I. Slepyan, Models and Phenomena in Fracture Mechanics. Berlin: Springer, 2002.

[14] M. Marder and S. Gross, "Origin of crack tip instabilities," Journal of the Mechanics and Physics of Solids, vol. 43, pp. 1-48, 1995.

[15] D. A. Kessler, "Steady-state cracks in viscoelastic lattice models," Physical Review E, vol. 59, no. 5, pp. 5154-5164, 1999.

[16] J. F. Lutsko, "Stress and elastic constants in anisotropic solids: Molecular dynamics techniques," Journal of Applied Physics, vol. 64, pp. 1152-1154, 1988.

[17] K. Ravi-Chandar, "Dynamic fracture," in Comprehensive Structural Integrity (I. Milne, R. O. Ritchie, and B. Karihaloo, eds.), vol. 2, ch. 5, Elsevier, 2003.

[18] J. R. Rice, Fracture, vol. 2, ch. Chapter 3, pp. 191-311. Academic Press, 1968. 